\title{
O EQUILÍBRIO FUNCIONAL DE INDIVÍDUOS COM DOENÇA DE PARKINSON EM TRATAMENTO COM FISIOTERAPIA EM GRUPO NO FORMATO DE CIRCUIT TRAINING
}

Fabiana Araújo Silva; Lúcia Martins Barbatto; Vanessa Niens Van Den Broek; Carla de Oliveira Carletti; Thiago Kendy Kawano; Tânia Cristina Bofi; Augusto Cesinando de Carvalho.

Universidade Estadual Paulista - UNESP, Departamento de Fisioterapia, Campus de Presidente Prudente, SP. E-mail: fabyana as@hotmail.com

\section{RESUMO}

Indivíduos com Doença de Parkinson tem dificuldades em modular as estratégias de equilíbrio. Os déficits de equilíbrio e a inabilidade postural, quando não tratadas, podem levar a aumento da frequência de quedas e fraturas. O terapêutica deve ser focada na prática de tarefas funcionais repetitivas, que podem melhorar ou manter o desempenho motor e a capacidade aeróbica. A Fisioterapia em Grupo no formato de Circuit Training (FGCT) utiliza exercícios funcionais específicos com foco na repetição e na progressão contínua de exercícios em um número de estações de trabalho organizadas em um formato de circuito. O objetivo deste estudo foi avaliar o equilíbrio funcional de indivíduos com Doença de Parkinson em tratamento com FGCT. Participaram do estudo 13 indivíduos com diagnóstico clínico de Doença de Parkinson. Este trabalho permite inferir que o protocolo terapêutico e o tempo utilizado foi capaz de manter o equilíbrio funcional, porém não foram capazes de modifica-lo.

Palavras - chave: fisioterapia, doença de Parkinson, exercício, exercícios em circuito, equilíbrio postural.

\section{THE FUNCTIONAL EQUILIBRIUM OF INDIVIDUALS WITH PARKINSON DISEASE IN TREATMENT WITH GROUP PHYSIOTHERAPY IN CIRCUIT TRAINING FORMAT}

\footnotetext{
ABSTRACT

People with Parkinson's disease have difficulty in modulating the equilibrium strategy. Balance deficits and postural disability, when untreated, can lead to increased frequency of falls and fractures. The therapy should be focused on the practice of repetitive functional tasks, which can improve or maintain engine performance and aerobic capacity. Physiotherapy Group in Circuit Training format (PGCTF) uses specific functional exercises focusing on repetition and continuous progression exercises in a number of workstations arranged in a circuit format. The aim of this study was to evaluate the functional balance of people with Parkinson's disease drugs in treatment PGCTF. The study enrolled 13 patients with clinical diagnosis of Parkinson's disease. This work allows us to infer that the therapeutic protocol and time used was able to maintain the functional balance, but were not able to modify it.

Keywords: Physical Therapy Specialty, Parkinson disease, exercise, circuit-based exercise, postural balance.
} 


\section{INTRODUÇÃO}

A Doença de Parkinson (DP) caracteriza-se por acometimento de neurônios dopaminérgicos resultando em desordens dos movimentos voluntários e automáticos. Os principais sintomas são tremor em repouso, bradicinesia, rigidez e inabilidade postural ${ }^{1}$.

Esses sintomas contribuem para alterações motoras que resultam em mudanças na marcha, dificuldade em realizar transferências, déficit de equilíbrio e maior risco de quedas. Essas alterações levam a um declínio da mobilidade e da participação social, contribuindo para a condição de sedentarismo ${ }^{2}$.

A degeneração dos núcleos da base geram um padrão inibitório exacerbado da via nigroestriatopalidal, o que faz os indivíduos com DP terem dificuldades em modular as estratégias de equilíbrio. Os déficits de equilíbrio e a inabilidade postural, se não tratados, podem levar a aumento da frequência de quedas e fraturas, contribuindo para o risco de comorbidades. Essas disfunções ocorrem normalmente nas fases médias ou mais avançadas da doença e são fatores pouco influenciados pelos medicamentos, necessitando de outras abordagens ${ }^{2}$.

Em geral, a combinação da farmacoterapia com programas de reabilitação é a estratégia de tratamento ideal para o controle dos sintomas. O uso do exercício físico visa melhorar e manter a facilidade e segurança das AVD, prevenir complicações secundárias e retardar a progressão da doença ${ }^{3,4}$.

O treinamento de indivíduos com DP deve ser focado em prática de tarefas funcionais repetitivas, que podem melhorar o desempenho motor e a capacidade aeróbica. Programas terapêuticos que necessitam de agilidade sensório-motora vêm sendo aplicados na DP, destacando-se exercícios que envolvem coordenação, equilíbrio, destreza e treino de marcha com variação da velocidade, associação de tarefas cognitivas e utilização de sequências mais desafiadoras ${ }^{4,5}$.

A Fisioterapia em Grupo no formato de Circuit Training (FGCT) é um modelo de fisioterapia que utiliza exercícios funcionais específicos realizados de forma intensiva com foco na repetição e progressão contínua em uma série de estações de trabalhos dispostos num formato de circuito dirigido, visando melhorar a mobilidade geral, a capacidade de andar, a coordenação e o equilíbrio funcional. Estudos demonstraram que a FGCT está associada a um maior grau de independência em diversas atividades e ainda a uma melhor satisfação no momento da alta da reabilitação do que a fisioterapia convencional individual $(\mathrm{FCl})^{6}$. A reabilitação em grupo auxilia na melhora do estado de saúde global do paciente e aumenta a socialização entre os indivíduos. Sendo assim esse trabalho objetivou avaliar o equilíbrio funcional de indivíduos com DPem tratamento com FGCT.

\section{METODOLOGIA}

Participaram deste trabalho 13 indivíduos com DP submetidos à FGCT. Os critérios de elegibilidade foram diagnóstico clínico de DP, estar entre o estágio 1 e 3 da escala de Hoehn e Yahr, ser capaz de realizar a marcha com ou sem auxílio de órtese e não apresentar déficits cognitivos avaliados pelo mini-exame do estado mental ${ }^{7,8}$. Todos os voluntários participantes foram informados sobre os objetivos e procedimentos do estudo e após concordarem com sua participação assinaram um termo de consentimento livre e esclarecido submetido ao Comitê de Ética da FCT (CAAE: 47863415.6.000.56402).

Antes de iniciar a FGCT os indivíduos foram submetidos a uma entrevista individual para coleta de dados e a seguir foi realizada a avaliação inicial (AV1) utilizando a Escala Modificada de Berg (EMG) e após 24 sessões de intervenção com FGCT foi realizada a avaliação final (AV2). Para análise dos dados foi utilizado o Teste $t$ dependente para amostras pareadas considerando o valor de $p \leq 0,05^{9}$. 
A FGCT ocorre no formato de estações, que corresponde a um determinado exercício, previamente montados em espaços diferentes e interligados. Os exercícios tem diferentes níveis de dificuldades, utilizando bastões, bolas, bambolês, cadeiras, escada e rampa, cones e mapas de sinalização. Cada indivíduo realiza o exercício na velocidade que consegue, porém é estimulado a realizar cada vez mais rápido para que o nível de dificuldade seja aumentado. Antes de iniciar a terapia é realizada a aferição da pressão arterial e cada indivíduo recebe um número correspondente a estação que ele deverá iniciar. Ele permanece por 2 minutos em cada estação e após este tempo, troca de estação. Durante a sessão, que tem duração de 60 minutos, ele passa por todas as estações, podendo ou não repetir algumas delas.

Antes de iniciar as estações, os indivíduos realizam alongamentos globais, realizados de forma ativa, orientados por um fisioterapeuta.

As estações são: 1ạ - Quatro marcações de $10 \mathrm{~cm}$ de diâmetro, colocadas no chão formando um quadrado. O paciente na posição ortostática segurando um bastão deve tocar as marcações, realizando rotação de tronco; 2a - Três marcações colocadas na parede a $150 \mathrm{~cm}$ de altura do chão, formando um triângulo. Na posição ortostática, o paciente deve tocar na marcação e em seguida tocar seu nariz, alternando os braços; 3a - Na posição sentada, o paciente deve segurar uma bola tamanho 10 com uma das mãos. Ele deve segurar a bola em uma das mãos e abduzir esse braço, em seguida encontra os braços na linha média, trocando a bola de mão, e abduzir o braço que está segurando a bola; 4a - Seis bambolês dispostos em pares, no chão. 0 paciente deve passar pelos bambolês colocando um pé dentro de cada bambolê, ao final caminha por fora dos bambolês retornando para o início; 5a - Três marcações de $10 \mathrm{~cm}$ de diâmetro posicionados em forma um triângulo, colados na parede a $220 \mathrm{~cm}$ do chão. Na posição ortostática e segurando um bastão com as mãos, o paciente deve tocar cada círculo com a outra extremidade do bastão; 6a - Na posição ortostática, o paciente segura uma bola tamanho 10 com uma das mãos e passa a bola para a outra quando as mãos se encontrarem na frente e atrás do tronco, de modo que a bola contorne sua cintura; 7a - Em pé, apoiando em uma barra de ação, o paciente deve realizar agachamentos; 8a - Sentado em uma cadeira, próximo a uma mesa onde está uma caixa com pequenos blocos de madeira. O paciente deve pegar um bloco e colocar do outro lado da caixa, alternando as mãos; 9a - Em uma escada rampa em forma de "L", com 5 degraus em um lado, uma plataforma plana de $(90 \times 90) \mathrm{cm}$ interligada com uma rampa de 6 graus de inclinação. O paciente deve subir pela escada e descer pela rampa e vice versa; 10 - Em um step retangular com altura de $15 \mathrm{~cm}$, posto no chão, onde o indivíduo deve realizar tríplice flexão com a perna, de modo a colocar alternadamente os pés sobre o mesmo. Estas sessões de FGCT ocorrem duas vezes por semana.

\section{RESULTADOS}

Foram avaliados 13 indivíduos parkinsonianos do sexo masculino com idade média de $73,15 \pm 5,52$ anos sendo 1 indivíduo no estágio zero, 6 no estágio um, 2 no estágio dois, 2 no dois e meio e 1 no terceiro estágio da Escala de Estágios de Incapacidade de Hoehn e Yahr.

A análise dos resultados revelou valores da EMB na AV1 de 50,63 $\pm 4,15$ pontos e 50,45 \pm 4,78 pontos na AV2. A análise estatística não demonstrou diferença entre as duas avaliações ( $t=$ 0,25 sendo $p=0,803$ ).

\section{DISCUSSÃO}

Sabe-se que nas fases iniciais da doença as desordens de equilíbrio são pouco presentes e pouco incapacitantes, agravando-se com a progressão da mesma. Em fases posteriores essas desordens passam a ser mais aparentes e incapacitantes, contribuindo para a inatividade e o aumento do risco de quedas. Isso pode estar relacionada com o declínio da eficácia do tratamento medicamentoso (10). No presente estudo os participantes estão classificados em estágios de 
Hoehn e Yahr entre 0 ao 3, estando a maioria (53\%) em estágios mais brandos da doença, nos quais as desordens de equilíbrio estão pouco presentes e consequentemente os exercícios utilizados na FGCT não apresentam efeitos importantes.

A maioria dos indivíduos com DP apresentam uma interação deficitária entre os sistemas neurais responsáveis pelo equilíbrio, pois existe um conflito no processamento sensitivo central devido exacerbação das reações vestíbulo-galvânicas, declínio visual e isto resulta em estratégias inadequadas para gerar estabilidade estática ou dinâmica consequentemente estes indivíduos tornam-se incapazes de gerarem movimentos compensatórios. Diante disto as estratégias de tratamento devem proporcionar atividades que favoreçam estímulos somatossensoriais e visuais, com o objetivo de gerarem estratégias motoras mais adequadas para as perturbações. A FGCT é um modelo que proporciona estes estímulos, todavia a maioria dos indivíduos deste estudo não apresentou déficit de equilíbrio importante e por isso pode-se inferir que a interação entre os sistemas neurais não estão em grau deficitário avançado.

Scalzo et al (2009) constatou uma correlação negativa do instrumento em relação ao tempo de diagnóstico da doença e uma correlação modera entre a classificação no estágio da doença pela escala de Hoehn-Yahr ${ }^{11}$. Em alguns estudos com essa população a média de pontos obtidos pelos participantes variaram entre 47,2 a 53,67 pontos, os resultados obtidos no presente estudo os valores de média obtidos foram de 50,63 para AV1 e 50,45 para AV2 ${ }^{12}$.

A reabilitação em grupo contribui na melhora do estado de saúde global do paciente, permitindo maior socialização entre os indivíduos. Programas terapêuticos que exploram a agilidade sensório-motora vêm sendo aplicados na DP. Tais programas envolvem atividades de equilíbrio, coordenação, destreza e treino de macha associado a tarefas cognitivas, realizadas de uma forma intensiva, com foco na repetição e progressão dos exercícios. Esse modelo fisioterapêutico tem como objetivo melhorar a mobilidade geral, a capacidade de andar, a coordenação e o equilíbrio funcional. Os indivíduos que são atendidos dentro desse modelo fisioterapêutico apresentaram-se bastante motivados e satisfeitos com a terapia, pois participaram efetivamente durante as tarefas propostas, além de proporcionar maior interação entre os participantes ${ }^{4,13}$.

Os resultados deste trabalho permite inferir que o tratamento com a FGCT é uma terapia segura, viável e aplicável, promovendo a oportunidade de realizar atividades ativas, evitando o sedentarismo, mantendo a funcionalidade e prevenindo complicações secundárias e incapacidades decorrentes da progressão da doença, além de proporcionar participação social.

\section{CONCLUSÃO}

O protocolo terapêutico e o tempo utilizado não foram capazes de modificar o equilíbrio funcional de indivíduos com DP submetidos a FGCT.

\section{REFERÊNCIAS}

Hallett M. Parkinson revisited: pathophysiology of motor signs. Advances in neurology. 2003;91:19-28. PubMed PMID: 12442661.

van den Heuvel MR, Kwakkel G, Beek PJ, Berendse HW, Daffertshofer A, van Wegen EE. Effects of augmented visual feedback during balance training in Parkinson's disease: a pilot randomized clinical trial. Parkinsonism \& related disorders. 2014 Dec;20(12):1352-8. PubMed PMID: 25283070.

Alves da Rocha P, McClelland J, Morris M. Complementary physical therapies for movement disorders in parkinson's disease: a systematic review. European journal of physical and rehabilitation medicine. 2015 Jul 3. PubMed PMID: 26138090. 
de Dreu MJ, van der Wilk AS, Poppe E, Kwakkel G, van Wegen EE. Rehabilitation, exercise therapy and music in patients with Parkinson's disease: a meta-analysis of the effects of music-based movement therapy on walking ability, balance and quality of life. Parkinsonism \& related disorders. 2012 Jan;18 Suppl 1:S114-9. PubMed PMID: 22166406.

Allen NE, Sherrington C, Suriyarachchi GD, Paul SS, Song J, Canning CG. Exercise and motor training in people with Parkinson's disease: a systematic review of participant characteristics, intervention delivery, retention rates, adherence, and adverse events in clinical trials. Parkinson's disease. 2012;2012:854328. PubMed PMID: 22191077. Pubmed Central PMCID: 3236465.

English CK, Hillier SL, Stiller KR, Warden-Flood A. Circuit class therapy versus individual physiotherapy sessions during inpatient stroke rehabilitation: a controlled trial. Archives of physical medicine and rehabilitation. 2007 Aug;88(8):955-63. PubMed PMID: 17678655.

Hoehn MM, Yahr MD. Parkinsonism: onset, progression, and mortality. 1967. Neurology. 2001 Nov;57(10 Suppl 3):S11-26. PubMed PMID: 11775596.

Cockrell JR, Folstein MF. Mini-Mental State Examination (MMSE). Psychopharmacology bulletin. 1988;24(4):689-92. PubMed PMID: 3249771.

Berg K, Wood-Dauphinee S, Williams Jl. The Balance Scale: reliability assessment with elderly residents and patients with an acute stroke. Scandinavian journal of rehabilitation medicine. 1995 Mar;27(1):27-36. PubMed PMID: 7792547.

Santens P, Boon P, Van Roost D, Caemaert J. The pathophysiology of motor symptoms in Parkinson's disease. Acta neurologica Belgica. 2003 Sep;103(3):129-34. PubMed PMID: 14626691.

Scalzo PL, Nova IC, Perracini MR, Sacramento DR, Cardoso F, Ferraz HB, et al. Validation of the Brazilian version of the Berg balance scale for patients with Parkinson's disease. Arquivos de neuro-psiquiatria. 2009 Sep;67(3B):831-5. PubMed PMID: 19838513.

Lun V, Pullan N, Labelle N, Adams C, Suchowersky O. Comparison of the effects of a self-supervised home exercise program with a physiotherapist-supervised exercise program on the motor symptoms of Parkinson's disease. Movement disorders : official journal of the Movement Disorder Society. 2005 Aug;20(8):971-5. PubMed PMID: 15838853.

Tomlinson CL, Patel S, Meek C, Herd CP, Clarke CE, Stowe R, et al. Physiotherapy intervention in Parkinson's disease: systematic review and meta-analysis. Bmj. 2012;345:e5004. PubMed PMID: 22867913. Pubmed Central PMCID: 3412755. https://doi.org/10.1136/bmj.e5004. 